\title{
L'indianisme au Brésil au travers des traductions, des adaptations et des transpositions en français du poème épique de José de Santa Rita Durão sur la découverte de Bahia
}

\author{
Caramurú. Poema épico do descobrimento da Bahia (1781)
}

\section{Alain Vuillemin}

Le passé contient-il l'avenir? L' “indianisme," la revendication d'une identité "indienne" spécifique qui s'est exprimée avec violence au Brésil, en juin 2013, contre des propriétaires terriens dans les États du Para et du Mato Grosso, est peut-être né dès les toutes premières rencontres entre les indiens Tupis et les Européens, dès la découverte du Brésil par Pedro Álvares Cabral, ${ }^{1}$ le 22 avril 1500. Telle paraît être la conviction qui semble animer à la fin du XVIIIème siècle un poète portugais né au Brésil, dans l'État du Minas Geras, le frère José de Santa Rita Durão, ${ }^{2}$ auteur d'une épopée en vers, en alexandrins, parue à Lisbonne, au Portugal, en 1781: Caramurú. Poema épico do descobrimento da Bahia 3 ("Caramuru. Poème épique sur la découverte de Bahia"). En langue tupi, "Caramurú”4 était le nom ou le surnom indien de Diogo Álvares

1 Pedro Álvares Cabral (vers 1467-1520), navigateur portugais, découvreur du Brésil le 22 avril 1500.

2 José de Santa Rita Durão (vers 1718-1784), né à Cata Preta (Mariana aujourd'hui), dans l'État des Minas Gerais, au Brésil, orateur et poète, ordonné prêtre dans l'ordre de Saint-Augustin et considéré comme le fondateur de l'indianisme au Brésil.

3 José de Santa Rita Durao, Caramurú. Poema épico do descobrimento da Bahia, Lisbóa (Portugal), na Regia officina typografica, 1781, in-12. Édition en ligne, site Scribd <http://www .scribd.com/api_user_11797_vivancrisologo/d/7289661-Jose-de-Santa-Rita-Durao -Caramuru-1781>, consulté le 28 juillet 2013.

4 Le mot "Caramurú" ("Caramuru" ou "Caramourou" selon les orthographes) désignerait en langue tupi les "murènes" ou les "dragons de la mer" auxquels les cadavres des naufragés étaient assimilés. Daniel Gavet et Philippe Boucher en proposent une autre interprétation dans la notice introductive de leur roman: "Caramurú" (orthographié "Caramourou" dans leur livre) signifierait "Homme de feu" en raison de la flamme et du bruit du mousquet que Diogo Álvares possédait. Pour José Santa Rita Durão, dans les réflexions qui précèdent son propre poème, "Caramurú" voudrait dire "Filho do trovão," le "Fils du Tonnerre," pour les mêmes raisons, le bruit et la flamme de la détonation. Pour Blaise Cendrars dans Brésil, des hommes sont venus, ce mot "Caramuru [...] signifie 'tombé dans le trou' en tupi." La signification exacte de ce surnom reste un sujet de controverses. Il faut voir sur ce point Antonio da

(C) ALAIN VUILLEMIN, 2015 | DOI 10.1163/9789004282087_017

This is an open access chapter distributed under the terms of the prevailing CC-BY-NC License at the time of publication. 
Correia, ${ }^{5}$ le tout premier habitant européen, portugais, du Brésil. Il aurait fait naufrage aux atterrages de Salvador da Bahia, à Rio Vermelho ou à proximité, sur la côte atlantique. Ce poème épique raconte son histoire et celle de son épouse indienne, Catarina Álvares Paraguaçú, ${ }^{6}$ venue en France, au temps du roi François Ier, entre $15^{26}$ et 1528 , et baptisée en Bretagne, à Saint-Malo, le 30 juillet 1528 , sous le nom de "Katherine du Brésil," 7 grâce à la protection du navigateur français Jacques Cartier ${ }^{8}$ et de son épouse, Catherine des Granches. ${ }^{9}$ Catarina Paraguaçú Álvares est devenue après sa mort, à Rio-de-Janeiro, en 1583, "A Mãe do Brasil," la “Mère du Brésil," comme l'a rappelé en 2001 un roman historique, Catarina Paraguaçu : A Mãe do Brasil, ${ }^{10}$ écrit par un auteur brésilien bahianais, Franco Tasso. ${ }^{11}$

Le récit de José de Santa Rita Durão a été traduit en français par Eugène Garay de Montglave ${ }^{12}$ en 1829, sous la forme d'un "roman-poème brésilien" en prose, intitulé Caramuru, ou la découverte de Bahia. ${ }^{13}$ Dès 1830, en France, un

Silva: Casamento do Paraguassu com o Filho do Fogo ["Le Mariage de Paraguassu avec le Fils du Feu"], Salvador (BA), sr, sd.

Diogo Álvares (ou Álvarez) Correia (vers 1474-1557), aventurier et navigateur portugais originaire de Viana de Castelo au Portugal, et premier européen à vivre au Brésil.

6 Catarina Álvares Paraguaçú (vers 1495-1583) dont le vrai nom aurait été "Guaibimpará" ("celle qui serait originaire de Guaibim," une localité près de Salvador da Bahia) et non "Paraguaçú" (qui signifierait la "grande eau," le "fleuve" ou la "haute mer," ou encore "celle qui a traversé la grande mer").

Un fac-simile du texte de ce certificat de baptême est reproduit en hors-texte dans Catherine du Brésil, filleule de Saint-Malo, Paris, Nouvelles Éditions Latines, 1948, 129.

8 Jacques Cartier (1491-1557), navigateur et explorateur français, découvreur du Canada en 1534 .

9 Catherine Des Granches, fille de Françoise Dumast et de Jacques-Honoré, chevalier et connétable de Saint-Malo.

10 Franco Tasso, Catarina Paraguaçú - A Mãe do Brasil ["Catarina Paraguaçú - la Mère du Brésil”], Rio-de-Janeiro (RJ), Editado pela Relume Dumará, 2001. Édition en ligne, sur le site Google Livres à <http://books.google.fr/books?id=PJMtAAAAYAAJ\&q=Catarina +Paragua $\% \mathrm{C}_{3} \% \mathrm{~A}_{7} \% \mathrm{C}_{3} \% \mathrm{BA}$. $+\mathrm{A}+\mathrm{M} \% \mathrm{C}_{3} \% \mathrm{~A}_{3} \mathrm{e}+$ do + Brasil, \&dq=Catarina+Paragua ${ }_{0} \mathrm{C}_{3} \%$ $\mathrm{A} 7 \% \mathrm{C}_{3} \% \mathrm{BA} .+\mathrm{A}+\mathrm{M} \% \mathrm{C}_{3} \% \mathrm{~A}_{3} \mathrm{e}+\mathrm{do}+\mathrm{Brasil}, \& \mathrm{hl}=$ fr\&sa=X\&ei=oZdcT9iPMMeA8wP-ncyDD w\&ved $=0$ CDoQ6AEwAA $>$, consulté le 28 juillet 2013 .

11 Franco Tasso Paes (né en 1946), journaliste et écrivain brésilien originaire de Bahia.

12 Eugène Garay de Montglave, de son vrai nom Eugène Moncla (1796-1878), polygraphe et traducteur, proche de Dom Pedro Ier (1798-1834), empereur du Brésil de 1822 à 1831, dont il a traduit la correspondance en français.

13 Voir Durão, José de Santa Rita: Caramaru, ou la découverte de Bahia [traduit par Eugène Garay de Montglave], Paris, Eugène Renduel, 1829.

Édition en ligne, site Gallica de la Bibliothèque Nationale de France: 
autre roman, Jakaré-Ouassou, ou les Tupinambas, ${ }^{14}$ écrit à deux mains par Daniel Gavet ${ }^{15}$ et par Philippe Boucher, ${ }^{16}$ reprenait le même sujet, avec un certain nombre de libertés. Le mythe a été entretenu en France et au Brésil à l'opéra, par un poème lyrique français, Paraguassú: chronique brésilienne ${ }^{17} \mathrm{de}$ J. O'Kelly et de J. Villeneuve, publié à Paris en 1855, et, au Brésil, à Rio-de-Janeiro, en 1892 , avec Moema, ${ }^{18}$ un autre opéra de Joaquim Torres Delgado de Carvalho ${ }^{19}$ dont le titre reprend le nom d'une des sœurs de Paraguaçú, "Moema," ${ }^{20}$ dont José de Santa Rita Durão prétend dans son Caramurú qu'elle se serait noyée par amour et par désespoir lors du départ de Diogo et de Paraguaçú pour la France. Le même mythe ressuscite en 1928 dans Macunaíma, ${ }^{21}$ un roman de Mario de Andrade $^{22}$ et l'un des textes fondateurs du modernisme brésilien. Il renaît encore en 1942, dans la littérature brésilienne, dans un récit écrit par Olga Obry, ${ }^{23}$ Catarina do Brasil : a Índia que descobriu a Europa, ${ }^{24}$ traduit en français par son auteur en 1948 sous le titre de Catherine du Brésil, filleule de

tome I: <http://gallica.bnf.fr/ark:/12148/bpt6k57403818.r=Santa+Rita+Durao.langFR>, tome II: <http://gallica.bnf.fr/ark:/12148/bpt6k5739362f.r=Santa+Rita+Durao.langFR>, tome III: <http://gallica.bnf.fr/ark:/12 148/bpt6k57403959.r=Santa+Rita+Durao.langFR>, consultés le 28 juillet 2013 .

14 Daniel Gavet et Philippe Boucher, Jakaré-Ouassou, ou les Tupinambas, Paris, Timothy Dehay, 1830, p. 237. Édition en ligne, site Gallica de la Bibliothèque Nationale de France. <http://gallica.bnf.fr/ark:/12148/bpt6k56272819.r=Jakar\%C3\%A9-Ouassou+ou+les+ Tupinambas.langFR $>$, consulté le 28 juillet 2013.

15 Daniel Gavet (1811-1883), polygraphe français et traducteur.

16 Philippe Boucher (1811-1885), pasteur protestant méthodiste français, proche de l'empereur du Brésil Dom Pedro Ier.

17 J. O'Kelly et J. Villeneuve, Paraguassú : chronique brésilienne, Paris, Bolle-Lassalle, 1855.

18 Delgado de Carvalho Joaquim Torres: Moema (opéra créé au Théâtre lyrique de Rio-de -Janeiro en 1892).

19 Joaquim Torres Delgado de Carvalho (1872-1921), compositeur et musicien brésilien.

20 Moema (?- vers 1526 ?) aurait été la sœur aînée de Paraguaçú, d'après José de Santa Rita Durão.

21 Mario de Andrade: Macunaíma : a herói sem nenhum caráter (Macounaïma: le héros sans aucun caractère, traduction française : 1997), São Paulo (SP), [Oficinas Gráficas de Eugenio Cupolo, 26 jul.] 1928.

22 Mario de Andrade (Mário Raul de Morais Andrade, 1893-1945), poète, romancier, musicologue, photographe et critique d'art brésilien, originaire de São Paulo.

23 Olga Obry (sr), romancière et traductrice franco-brésilienne.

24 Olga Obry, Catarina do Brasil: a Índia que Descobriu a Europa [ « Catherine du Brésil:I'indienne qui découvrit l'Europe »], Rio-de-Janeiro (RJ), Atlantica Editora, 1942. Édition en ligne, site Google Livres à<http://books.google.fr/books/about/Catarina_do_Brasil_a_\%C $3 \%$ ADndia_ que_descobri.html?id=S6gXAAAAIAAJ\&redir_esc=y>, consultéle 28 juillet 2013. 
Saint-Malo. ${ }^{25}$ Un film brésilien de Guel Arraes, ${ }^{26}$ sorti en 2001, Caramurú. A invenção do Brasil, ${ }^{27}$ a retracé cette histoire, de même qu'un roman, déjà cité et publié aussi en 2001, Catarina Paraguaçú. A Măe do Brasil de Franco Tasso, qui n'avait pas encore été traduit en français en 2013. Ces textes et ces films décrivent comment le monde connu s'est brusquement étendu en 1500, au temps des grandes découvertes, à la fois pour les indiens Tupis du Brésil et pour les Européens. Deux civilisations, deux univers, se sont rencontrées et se sont aussi affrontées. Si on entend par "mondialisation," en cet exemple précis, la création d'un espace et d'une entité politique et culturelle unique par le biais de la conquête du Brésil par le Portugal, ces écrits conçus entre la fin du XVIII siècle et le début du XXI siècle reconstruisent une sorte d'archéologie de cette unification et de cette "globalisation," pour s'exprimer en des termes qui ne sont apparus dans la langue française que vers la fin du XX siècle. La constitution au XVI siècle des grands empires coloniaux européens a été une préfiguration de ce phénomène. Ce processus a aussi engendré sa propre négation. L'évolution des titres cités est déjà une indication. Entre le poème épique de José de Santa Rita Durão et les derniers romans d'Olga Obry et de Franco Tasso, la perspective initiale, coloniale et "européaniste," s'inverse presque complètement : l' "indianisme," la prise de conscience d'un sentiment identitaire propre, indien et indigéniste, purement brésilien, s'affirme avec de plus en plus de force. Ce que ces traductions et ces transpositions en langue française révèlent aussi, c'est que cette aspiration est très mêlée. Ces livres racontent la naissance d'une identité métisse, hybride. Comment se concilient en ces témoignages composites la revendication d'un héritage autochtone, la reconnaissance d'une multiplicité d'apports étrangers, et le désir de les assimiler, de se les approprier et de les fondre ensemble?

\section{Un héritage autochtone}

L'indianisme, c'est d'abord un héritage autochtone, indigène, qui est réhabilité et revendiqué. C'est le patrimoine des anciens indiens Tupis ou Tupinambas qui habitaient dans la région de Bahia et qui étaient présents tout au long des

25 Olga Obry, Catherine du Brésil, filleule de Saint-Malo, (Paris, Nouvelles Éditions Latines, 1948). Édition en ligne, site Google Livres à <http://books.google.fr/books?id=xYkerLo7Df MC\&pg=PA165\&lpg=PA165\&dq=Bapt $\% \mathrm{C}_{3} \% A A m e+d e+C a t h e r i n e+d u+B r \% C_{3} \% A g s i l \& s$ ource =bl\&ots =zecupmCVI $5 \&$ sig $=$ YdQeZf8eISK ${ }_{52}$ BH8OadlbXvn_J4\&hl=fr\&sa $=X \&$ ei $=$ nJFcT-XjCMXq8QOEyvWvBw\&ved=oCDkQ6AEwAA\#>, consulté le 28 juillet 2013 .

26 Guel Arraes (Miguel Arraes de Alencar Filho), né en 1953, cinéaste brésilien originaire de Recife, Pernambuco.

27 Guel Arraes, Caramurú. A invenção do Brasil («Caramurú. La découverte du Brésil»), Globo Filmes-Lereby Productions, 2001. 
côtes du Brésil, de l'embouchure de l'Amazone à l'Uruguay actuel. Ce sont leurs us et leurs coutumes traditionnelles, leurs croyances et leurs convictions ancestrales et, aussi, leurs rites, y compris les plus cruels, qui sont rappelés.

La description des coutumes et de la manière de vivre des indiens Tupis avant l'arrivée des Européens est une première affirmation identitaire, une première forme d'indianisme. Ce qui est dit est toutefois très reconstruit, deux ou trois siècles plus tard, à travers ce que les auteurs différents qui se sont intéressés à la légende de Diogo Álvares et de Catherine Paraguaçú, au Brésil et en France, ont pu avoir lu ou retenu de témoignages contemporains de la découverte du Brésil au XVI siècle, à savoir : Nus, féroces et anthropophages ${ }^{28}$ de Hans Staden ${ }^{29}$ en 1557 et Les Singularités de la France antarctique ${ }^{30}$ d'André Thevet ${ }^{31}$ en 1558, et l'Histoire d'un voyage en la terre du Brésil, autrement dit Amérique ${ }^{32}$ de Jean de Léry ${ }^{33}$ en 1578 et, également, le chapitre "Des Cannibales" au premier livre des Essais $^{34}$ de Michel de Montaigne ${ }^{35}$ en ${ }^{1580-1587 . ~ A l f r e d ~}$

28 Hans Staden, Nus, féroces et anthropophages (1557) [ "Warhafftige Historia unnd Beschreibung einer Landtschafft der wilden, nacketen, grimmigen Menschfresser Leuthen... », traduit de l'allemand par Henri Ternaux Compans]. (Paris: Métailié, 2005).

29 Hans Staden (1525?-1576 ?), soldat et mercenaire d'origine allemande, embarqué comme canonnier en 1554 sur un vaisseau espagnol qui s'échoua près de l'île de Saint-Vincent, au large du Brésil. Capturé par une tribu Tupinambá qui pratiquait l'anthropophagie rituelle, il fut racheté à ces indiens par le capitaine d'un navire français après neuf mois de captivité.

30 André Thevet, Les Singularitez de la France antarctique, autrement nommée Amérique, \& de plusieurs terres et isles découvertes de nostre temps, Paris: chez les héritiers de Maurice de La Porte, 1558. [Éd. intégrale établie, présentée \& annotée par Frank Lestringant]. (Paris: Chandeigne, 1997). Édition en ligne, site Gallica: <http://gallica.bnf.fr/ark:/12148/ bpt6k109516t>, consulté le 28 juillet 2013.

31 André Thevet (1516-1590), prêtre franciscain français, chapelain de la flotte de l'amiral Nicolas Durand de Villegaignon, fondateur de la ville de Rio-de-Janeiro.

32 Jean de Léry, Histoire d'un voyage fait en la terre du Brésil, autrement dit Amérique (Première édition : (s. l.), pour A. Chuppin, 1578, In-12). Édition de Frank Lestringant. (Montpellier/ Presses du Languedoc/Max Chaleil Éditeur, 1992).

33 Jean de Léry (vers 1536-1613), voyageur et écrivain français, converti à la religion réformée, protestante, et cosmographe du roi de France Henri II.

34 Michel de Montaigne, Essais, 1580-1587 [traduction en français moderne du texte de l'édition de 1595 par Guy de Pernon]. (Leogeats: G. de Pernon, 2008). Édition en ligne, site Trismégiste : <http://www.bribes.org/trismegiste/montable.htm>, consulté le 28 juillet 2013. Édition en ligne, site Gallica:

- Tome I: <http://gallica.bnf.fr/ark:/12148/bpt6k6382240z.r=montaigne+essais.langFR>,

- Tome II: <http://gallica.bnf.fr/ark:/12148/bpt6k63435243.r=montaigne+essais.langFR>,

- Tome III: <http://gallica.bnf.fr/ark:/12148/bpt6k6343523p.r=montaigne+essais.langFR>,

- Tome IV: <http://gallica.bnf.fr/ark:/12148/bpt6k5780127w.r=montaigne+essais.langFR>,

- consultés le 28 juillet 2013 .

Michel de Montaigne (1533-1592), moraliste et philosophe français. 
Métraux ${ }^{36}$ y ajoute dans sa préface au roman d'Olga Obry, Catherine du Brésil, filleule de Saint-Malo, le nom d'Yves d'Évreux, ${ }^{37}$ un moine capucin français, auteur en 1615 d'une Suite de l'histoire des choses les plus mémorables en Maragan en $1613-1614,{ }^{38}$ à propos de la fondation de la ville de São Luis de Maranhâo, en 1612, dans l'État actuel du Maranhão, au nord-est du Brésil, où il s'était rendu entre 1612 et 1614, à la demande de son Ordre. On pourrait y ajouter le témoignage d'un autre missionnaire capucin, Claude d'Abbeville, ${ }^{39}$ Histoire de la mission des pères Capucins en l'Isle de Maragnan et terres circonvoisines, ${ }^{40}$ paru en 1614, qui évoque les mêmes singularités des mœurs indiennes.

Dans les traductions et les adaptations de Caramurú, le poème épique de José de Santa Rita Durão, les auteurs insistent surtout sur le caractère étrange, très exotique, des mœurs des indiens. Des préjugés moraux insidieux et contradictoires, accentuent les différences et forcent les traits. Dans Caramurú ou la découverte de Bahia, Eugène Garay de Montglave essaie de préserver le regard apparemment neuf, étonné que le jeune Diogo Álvares porte sur les us et les mœurs des indiens Tupis, tels que José de Santa Rita Durão les avait imaginés. Le chapitre VII de la traduction, intitulé "Un village américain," en décrit le mode de vie. Un chef indien, Gupeva, explique à Diogo Álvares comment les grandes étapes de l'existence se déroulent pour les siens, pour les membres de sa tribu. L'habitat est collectif. La vie est communautaire. Le village se compose de huit grandes cabanes en bois, en osier et en roseaux, disposées en carré. Chacune de ces constructions abrite entre six cents et mille personnes. Á l'intérieur, à chaque pas, des hamacs se balancent. C'est là que les indiens de Gupeva naissent, vivent, grandissent, mangent, dorment, méditent et meurent, le moment venu. Leurs joies et leurs peines, leurs souffrances et leurs deuils, leurs rites et leurs cérémonies, leurs fêtes et leurs danses, leurs libations et

$36 \quad$ Alfred Métraux (1902-1963), anthropologue d'origine suisse, naturalisé américain en 1941.

37 Simon Michellet, en religion Yves d'Évreux (vers 1570-vers 1633), moine capucin français, historien et explorateur français.

38 Yves d'Évreux, Suite de l'histoire des choses plus mémorables advenues en Maragnan ès années 1613 et 1614. Second traité [Texte imprimé]. (Par F. Yves d'Évreux.). Paris: F. Huby, MCDXC (sic) [édition critique du texte complet par Franz Obermeier, Kiel: WestenseeVerlag, cop. 2012].

39 Clément Foullon, en religion Claude d'Abbeville (?-1632), moine capucin français, missionnaire au Brésil en 1612.

40 Claude d'Abbeville, Histoire de la mission des Pères capucins en l'isle de Maragnan et terres ciconvoisines, où est traicté des singularitez admirables et des moeurs merveilleuses des Indiens habitans de ce pays, avec les missives et advis qui ont esté envoyez de nouveau par le R.P. Claude d'Abbeville. (Paris: imprimerie de F. Huby, 1614). 
leurs festins. Tous vivent nus. Le film de Nelson Pereira dos Santos, ${ }^{41}$ Como gostoso era meu francês ${ }^{42}$ en est une illustration très exacte. Le roman d'Olga Obry, Catherine du Brésil, filleule de Saint-Malo, insiste sur cet état naturel très primitif. La nudité paraît avoir été la plus grande différence par rapport aux mours européennes.

Les convictions ancestrales, religieuses et morales, des indiens Tupis sont remémorées. Caramurú, le poème épique de José de Santa Rita Durão, en reste la source principale. Olga Obry y ajoute d'autres sources dans Catherine $d u$ Brésil, filleule de Saint-Malo, en un appendice intitulé "Á la recherche d'une trace perdue," Gupeva expose ces croyances à Diogo Álvares au chant III de l'épopée de José de Santa Rita Durão et aux chapitres IX, X et XI de la transposition d'Eugène Garay de Montglave. Olga Obry les évoque d'une manière très allusive en la première et en la seconde partie de son propre roman, avant que Diogo Álvares ne fasse intrusion dans la vie de la future Catarina-Paraguaçú. Les indiens Tupis auraient cru en l'existence d'un Être suprême, un "Être puissant qui a créé le monde,"43 qui aurait régi le ciel, commandé à la pluie, à la grêle, aux vents, à la tempête. Il aurait été le maître des nuées, des éclairs et de la foudre. Il aurait habité le ciel. Il aurait été "l'architecte du monde," 44 un être créateur, démiurgique, qui aurait vaincu le néant, créé le ciel, la terre, les ténèbres et la lumière. Les indiens Tupis l'auraient appelé le grand "Tupa" ${ }^{55} \mathrm{ou}$ "Tupan" (le "Tonnerre") mais cet être, Gupeva en fait l'aveu, serait demeuré "incompréhensible," 46 énigmatique. Son œuvre serait restée inachevée. Cet "Être suprême" n'aurait pas encore enseigné aux indiens Tupis comment résister au mal. Le monde serait encore infesté d' "Anhangás,"47 de créatures malfaisantes, maléfiques, enfermées par un arrêt du grand Tupa en un endroit, par-delà les montagnes, où brûlerait "un incendie que le temps n'éteindra point."48 Á l'inverse, par-delà ces mêmes montagnes, il existerait aussi une "contrée de bonheur [...], un jardin sans modèle,"49 un lieu délicieux où, au sein du grand Tupa, vivraient les âmes de ceux qui furent des justes durant

\footnotetext{
41 Nelson Pereira dos Santos, né en 1928, cinéaste brésilien originaire de São Paulo (SP).

42 Nelson Pereira dos Santos, Como gostoso era meu francês («Qu’il était bon mon petit Français »), long métrage, DVD, 1971.

43 José de Santa Rita Durao, Caramaru, ou la découverte de Bahia [traduit par Eugène Garay de Montglave], (Paris, Eugène Renduel, 1829, tome I, 69).

44 Durão, tome I, 69.

45 Durão, tome I, 69.

46 Durão, tome I, 167.

47 Durão, tome I, 180.

48 Durão, tome I, 180.

49 Durão, tome I, 182.
} 
leurs vies terrestres. Bref, aux indiens Tupis, il n’aurait manqué que la révélation. Ils auraient déjà entrevu Dieu, le Paradis, l'Éden et l'Enfer. L'exposé de ces croyances attribuées aux indiens primitifs est très réfracté par les convictions religieuses, chrétiennes et catholiques, du frère José de Santa Rita Durão, professeur de théologie à l'université de Coïmbra, au Portugal, à l'époque où il concevait son poème épique à la gloire de Caramurú.

En ces lieux, à cette époque, au tout début du XVI siècle, la nature était violente et les mœurs aussi. Dans Catherine du Brésil, filleule de Saint-Malo d'Olga Obry dont l'action se déroule là où "le monde finit [et] où le soleil se lève," ${ }^{50}$ les cinq occas (de longues cabanes droites) disposées en cercle qui forment le taba, le village natal de l'hérö̈ne, "Paraguassu," dans le récit, sont entourés de pieux pointus qui servaient de protection contre les assauts des tribus voisines. Les guerres étaient fréquentes. "Des crânes fichés sur de hauts piquets," raconte Olga Obry, "prouvent que [des] ennemis ont eu à payer les frais [de] luttes récentes, et le dernier prisonnier achève d'engraisser sous les soins experts des femmes de la taba." ${ }^{\prime 51}$ Como Gostoso era meu francês, le film de Nelson Pereira dos Santos, reproduit avec beaucoup de fidélité et de précision ces mœurs guerrières des indiens Tupis à partir de ce que José de Santa Rita Durão raconte aux chants III et IV de Caramurú, et qu'Eugène Garay de Montglave ou Daniel Garet et Philippe Boucher décrivent dansJakaré-Ouassou, ou les Tupinambas. En ce passage, Diogo Álvares intervient, en armure, armé d'un mousquet, dans une guerre locale entre indiens, aux côtés des Tupinambas de Gupeva, aidés par Taparica, une reine indienne, accompagnée de mille amazones et de six mille autres guerriers. En face, les ennemis sont menés par un chef terrible, Jaracaca, le chef des indiens Caetés alliés à d'autres tribus, les Morgates, les Ovecates, les Maques, les Petiguares, les Agirapirangas, les Itatis, les Tapuias. Les combats sont féroces. Les affrontements, au corps à corps, sont sans pitié. Les femmes indiennes y participent, achevant les blessés à coups de massue. Les prisonniers vaincus sont dévorés par les vainqueurs. C'est très exactement le sort que subit le premier gouverneur portugais de Bahia, Francisco Pereira Coutinho, avec ses soldats, en 1547, dans la réalité, comme le raconte Jakaré-Ouassou, ou les Tupinambas de Daniel Garet et de Philippe Boucher. Ces indiens étaient des anthropophages. Sans un mousquet miraculeusement sauvé lors de son naufrage, Diogo Álvares aurait connu le même sort. La détonation de ce mousquet aurait terrifié les indiens qui s'apprêtaient à lui faire subir un mauvais sort. Diogo Álvares aurait été alors adoré par les indiens Tupis come un dieu vivant pour cette raison. Les témoignages de Hans

50 Obry, Catherine du Brésil, 13.

51 Obry, Catherine du Brésil, 13-14. 
Staden dans Nus, féroces et anthropophages, d'André Thévet dans Les Singularités de la France antarctique et de Jean de Léry dans son Histoire d'un voyage fait en la terre du Brésil corroborent ces pratiques guerrières. Olga Obry se contente d'ajouter dans Catherine du Brésil, filleule de Saint-Malo une restriction: "si les Tupinambas étaient anthropophages, ils ne mangeaient que leurs ennemis - par tradition et vengeance - jamais leurs amis."52 Telle aurait été leur morale. Par contre, pour José de Santa Rita Durão, traduit par Eugène Garay de Montglave, ces indiens étaient de "féroces anthropophages, ennemis de tout pouvoir"53 qu'il convenait de civiliser, comme en conviennent Duplessis, le capitaine français qui ramène Catherine et Diogo Álvares vers le Brésil, et Diogo Álvares lui-même. Ces "autres," si cruels, si barbares, si radicalement différents, devaient devenir des semblables, des êtres policés, européanisés, identiques ou presque.

En ces transpositions et en ces adaptations de l'histoire de Caramurú et de Paraguaçú - Diogo Álvares et Catherine Álvares - l'indianisme," c'est tout d'abord la prise de conscience de la persistance au Brésil d'un héritage autochtone, indien ou plutôt amérindien et brésilien, constitué par le souvenir de coutumes traditionnelles, de croyances anciennes et de pratiques guerrières particulièrement cruelles et féroces. Ce legs est très ambigu. Dans Caramuru, ou la découverte de Bahia d'Eugène Garay de Montglave, Diogo Álvares découvre cette indianité avec étonnement. Dans Catherine du Brésil, filleule de SaintMalo d'Olga Obry, l'histoire de cette indienne, cette Paraguaçú qui découvrit l'Europe, l'étonnement paraît avoir été réciproque. La première rencontre entre Paraguaçú et Diogo Álvares fut une double découverte réciproque. Ce fut aussi un terrible choc des civilisations.

\section{Les apports étrangers}

En cette indianité revendiquée, il est des apports étrangers. Paraguaçú, devenue Catherine du Brésil après son baptême en France, en a une vision prémonitoire à bord du navire du capitaine Duplessis qui la ramène au Brésil. Au chant IX du poème de José de Santa Rita Durão et aux chapitres 26 à 30 du roman d'Eugène Garay de Montglave, la jeune femme entre en extase. Elle entrevoit alors l'histoire du Brésil telle qu'elle se déroulera du milieu du XVI siècle jusqu'à la fin du XVIII siècle. Elle décrit aussi ce que les contacts avec

\footnotetext{
52 Obry, Catherine du Brésil, 17.

53 José de Santa Rita Durão, Caramaru, ou la découverte de Bahia [traduit par Eugène Garay de Montglave], Paris, Eugène Renduel, 1829, tome 2, 214.
} 
l'Europe apporteront au Brésil, à savoir la foi chrétienne, la civilisation et, aussi des pratiques coloniales très brutales.

La foi chrétienne serait le premier de ces apports. C'est la conviction de José de Santa Rita Durão, élève des jésuites à Rio-de-Janeiro, prêtre dans l'ordre de Saint-Augustin puis professeur de théologie catholique à l'université de Coïmbra au Portugal. La traduction de son Caramurú par Eugène Garay de Montglave respecte ces certitudes. Dans Jakaré-Ouassou, ou les Tupinambas, l'attitude de Daniel Gavet et de Philippe Boucher est plus nuancée. Philippe Boucher était un pasteur protestant. Dans Catherine du Brésil, filleule de SaintMalo, le regard d'Olga Obry est beaucoup plus laïc. Dans Caramurú de José de Santa Rita Durão, en dépit du titre, le véritable sujet, le plus développé, c'est l'histoire de Paraguaçú, celle de la "Mère de la Patrie" ("À Mãe da Pátria") ${ }^{54}$ comme il le déclare au début du chant I de l'épopée, en une strophe qui n'a pas été traduite par Eugène Garay de Montglave. Le chant III rapporte les circonstances de la première rencontre entre Paraguaçú et Diogo Álvares, et les chants VI à X retracent le grand voyage en France, sous le règne supposé du roi Henri II, vers 1547. C'est une sorte de récit autobiographique qui raconte surtout comme la jeune indienne Tupi aurait découvert l'Europe, la France, Paris et le palais du Louvres, et les circonstances d'un baptême parfaitement imaginaire, au chant VII, en la présence du "monarque très chrétien Henri II de France [...] ami de la foi," ${ }^{55}$ avec, à ses côtés, la reine Catherine de Médicis. Diogo et son épouse

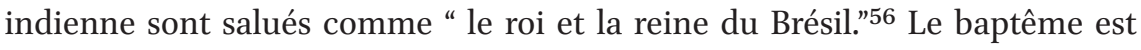
aussitôt décidé. La reine donne "son propre nom à Paraguaçú [et] veut que désormais on ne l'appelle plus que Catherine. ${ }^{.57}$ La légende se substitue à l'histoire. Olga Obry le relève en l'appendice à son propre roman, Catherine du Brésil, filleule de Saint-Malo. Au terme de Caramurú de José de Santa Rita Durão, au dernier chant, à la strophe LIX, Catherine Paraguaçú appelle les indiens Tupis à se soumettre à l'autorité du roi du Portugal et à se convertir à la religion de "ce Dieu puissant [Jésus-Christ] qui, attaché à une croix immortelle, expia vos crimes et paya votre perversité." ${ }^{58}$ Le propos est pieux. L'intention est édifiante. L’union de Catherine Paraguaçú et de Diogo Álvares

54 José de Santa Rita Durão, Caramurú. Poema épico do descobrimento da Bahia, Lisbóa, na Regia officina typografica, 1781, in-12. strophe 6. Version électronique sur le site Scribdà <http://www.scribd.com/api_user_11797_vivancrisologo/d/7289661-Jose-de-Santa-Rita-Du rao-Caramuru-1781>, consulté le 28 juillet 2013 .

55 José de Santa Rita Durão, Caramaru, ou la découverte de Bahia [traduit par Eugène Garay de Montglave], Paris, Eugène Renduel, 1829, tome II, 70.

56 Durão, tome II, 70.

57 Durão, tome II, 71.

$5^{8}$ Durão, tome II, 112. 
symbolise cette adhésion et cette conversion. La réalité fut toute autre. Daniel Gavet et Philippe Boucher le laissent entendre dans Jakaré-Ouassou, ou les Tupinambas dont l'action se situe aussi vers 1547 et où les indiens Tupis restent très attachés à leurs croyances traditionnelles. Dans Catherine du Brésil, filleule de Saint-Malo, Olga Obry imagine également que Catherine Álvares aurait déploré, en sa vieillesse, entre 1557 et 1586, la manière dont les indiens auraient été évangélisés avec beaucoup de rudesse et, aussi, refoulés non sans brutalité vers l'intérieur des terres.

La civilisation, le progrès des mœurs, des idées et des connaissances, est le second bienfait que la colonisation européenne aurait apporté aux indiens du Brésil. Les chants VIII et IX du Caramurú de José de Santa Rita Durão qui correspondent au troisième livre et aux chapitres 26 à 32 du roman poème d'Eugène Garay de Montglave, Caramurú ou la découverte de Bahia décrivent une vision prophétique qui est prêtée à Catherine Álvares Paraguaçú. Alors que le navire français qui la ramène vers le Brésil se trouve non loin de l'équateur, "l'innocente Paraguaçú, la modeste Catherine" 59 comme le commente Eugène Garay de Montglave, tombe soudain en extase et demeure plusieurs heures évanouie. Á son réveil, elle décrit les songes merveilleux que le Ciel lui aurait envoyés. Le ton est très emphatique. Sur un "vaste globe [terrestre] du plus pur diamant," ${ }^{\circ 0}$ elle aurait vu sa "belle patrie, ce Brésil magnifique [...], l'admirable rade de Bahia [puis une] magnifique cité qui se dessinait en amphithéâtre,"61 une forteresse à l'entrée de la barre, une autre sur les hauteurs et huit citadelles toute autour. Elle voit un "temple magnifique [...], un vaste palais."62 Elle aurait vu aussi, poursuit-elle, "mille événements, divers qui doivent jalonner le cours des siècles [...], surgir de provinces puissantes [...], de magnifiques cités," ${ }^{\prime 3}$ et elle aurait aussi pressenti la future indépendance du "Brésil échappant à son tour aux chaînes de la Lusitanie." ${ }^{44}$ Le Brésil ne deviendra indépendant qu'en 1822. En 1781, José de Santa Rita Durão ne pouvait en avoir qu'un pressentiment. Les premières strophes du chant VIII de l'épopée sont au contraire un éloge de la colonisation européenne. Duplessis, le capitaine du navire qui a mené Diogo Álvares jusqu'en France, lui propose l'aide du roi de France dans son "généreux projet d'instruire la rudesse de ces sauvages [les indiens Tupis],

\footnotetext{
59 Durão, tome II, 84.

60 Durão, tome II, 102.

61 Durão, tome II, 80.

62 José de Santa Rita Durão, Caramaru, ou la découverte de Bahia [traduit par Eugène Garay de Montglave], (Paris, Eugène Renduel, 1829, tome III, 9).

63 Durão, tome III, 98.

64 Durão, tome III, 97.
} 
et d'adoucir leur cruelle inhumanité."65 Diogo Álvares, toujours fidèle à sa patrie, se récrie. Il reste portugais du fond de l'âme s'il est convaincu que, "quand un peuple vit hors de la loi commune des nations, en proie au désordre et à l'anarchie, le droit de le soumettre, de l'éclairer, de le rendre humain, est le partage du premier occupant. Cette vérité nous est suggérée par la nature ellemême,"66 et il ajoute un argument en forme de syllogisme : "La nature a inspiré à Cabral l'heureuse idée d'aborder nos rivages. Cabral est portugais. Ces rivages appartiendront au Portugal." ${ }^{\prime 7}$ Les visions surnaturelles prêtées à Catarina Álvares Paraguaçú ne sont qu'une surenchère. La civilisation, le progrès, aurait été le plus grand apport de l'Europe et du Portugal au Brésil.

Les pratiques coloniales ont été un autre apport, très ambigu. Olga Obry affirme dans Catherine du Brésil, filleule de Saint-Malo, que "la colonisation du Brésil par les Portugais est, dans l'Histoire des Découvertes, un fait exceptionnel : elle fut en grande partie empreinte d'humanité [...]. Certes, il a eu des luttes, des révoltes, des représailles, des trahisons, des tueries [...] mais l'essentiel [...] c'était la naissance d'une nation dans le respect mutuel de la personne humaine, c'était la compréhension et la coopération entre individus de races différentes." ${ }^{\prime 8} \mathrm{Au}$ XVI siècle, laissent entendre Daniel Gavet et Philippe Boucher dans Jakaré-Ouassou, ou les Tupinambas, la réalité aurait été très différente. Francisco Pereira Coutinho, le premier donataire de la capitainerie de Bahia, arrivé à Salvador en 1536, ne serait venu que pour "asservir, enchaîner, et non pas civiliser ni convertir,"69 comme l'écrivent ces deux auteurs dans la "notice" qui sert d'introduction à leur roman. Ils le présentent comme un "barbare suzerain qui règne par le crime sur l'établissement portugais de Salvador." ${ }^{\prime 70}$ Un chef indien, Jakaré-Ouassou (le "grand crocodile" en langue Tupi) se révolte contre les exactions du gouverneur portugais. Caramurú (Diogo Álvares) est un témoin vieilli de ces événements. En 1547, la caravelle qui transporte Coutinho avec ses soldats s'échoue sur des bas-fonds de l'île d'Itaparica, au large de la ville de Salvador. Les naufragés sont assaillis par les guerriers de Jakaré-Ouassou. Sous le nombre, les Portugais succombent, Coutinho est tué, décapité puis dévoré rituellement. Á la fin du livre, les guerriers Tupinambas, réunis dans une forêt, chantent cette histoire, avant de se réfugier plus loin vers l'intérieur des terres. Ils sont désespérés. Ils se lamentent

\footnotetext{
65 Durão, tome III, 212.

66 Durão, tome III, 215.

67 Durão, tome III, 215 .

68 Obry, Catherine du Brésil, 146.

69 Daniel Gavet et Philippe Boucher, Jakaré-Ouassou, ou les Tupinambas. Paris: Timothy Dehay, 1830,33 .

70 Gavet, 33 .
} 
sur la grandeur passée et la puissance disparue des Tupinambas. Ils "ne savent point où ils vont. Ils marchent, ils pleurent," ${ }^{11}$ dit Olga Obry. La romancière donne une indication supplémentaire sur leur sort dans l'appendice historique de son roman, dans Catherine du Brésil, filleule de Saint-Malo: "les indiens, décimés par les misères et les épidémies, se [retirèrent] de plus en plus dans la sylve [la forêt], s'éloignant toujours davantage de la cote [...mais] le dieu du 'mota' [de la brousse, qui] attire les Indiens [...] les tue d'une mort lente et cruelle: disette de vivres, bêtes féroces, climat exténuant."72 Lauteure se contente de relever ces faits. C'est un chapitre douloureux de l'histoire du Brésil. Selon les estimations avancées, les peuples indigènes du Brésil auraient compté entre quinze et vingt millions d'individus en 1500. En un siècle, du fait des maladies et des épidémies, l'extermination fut massive : en 1600, moins de dix pour cent de ces populations auraient subsisté. Les revendications indianistes modernes se nourrissent aussi de ce terrible héritage du passé.

En 1781, dans son Caramurú, affirme José de Santa Rita Durão, Diogo Álvares serait venu "chercher en France les lumières qui lui paraissaient indispensables pour arracher les barbares [les peuples indiens] à leurs funestes erreurs." ${ }^{73} \mathrm{La}$ pénétration européenne a certes apporté au Brésil et aux indiens la foi, la religion chrétienne et la civilisation, une transformation profonde de la société, mais aussi des pratiques coloniales particulièrement brutales et violentes que Daniel Gavet et Philippe Boucher dénoncent dans Jakaré-Ouassou ou les Tupinambas, en prenant le parti des indiens Tupis. En cette indianité primitive que ces récits prétendent décrire, les apports étrangers sont très ambigus. Mais, comme l'observait l'anthropologue Alfred Métreaux, préfacier du roman d'Olga Obry, Catherine du Brésil, filleule de Saint-Malo, en 1953, ces traits contradictoires ont déterminé "le destin du Brésil." ${ }^{\text {4 }}$ Ces apports étrangers ont contribué à élaborer son identité mêlée.

\section{Une identité métisse}

Telle que José de Santa Rita Durão et Eugène Garay de Montglave la rapportent, et telle qu'Olga Obry la reprend en la fouillant, plus d'un siècle après, la légende de Diogo Álvares et de Catarina Paraguaçú est le récit d'une double découverte,

\footnotetext{
71 Obry, Catherine du Brésil, 39.

72 Gavet, 384 .

73 José de Santa Rita Durão, Caramaru ou la découverte de Bahia [traduit par Eugène Garay de Montglave]. Paris: Eugène Renduel, 1829, tome III, 211-212.

74 Alfred Métaux, "Préface." Catherine du Brésil. Paris: Nouvelles Éditions Latines, 1948, 11.
} 
mutuelle, celle de l'autre, celle de celui qui est un étranger. Á travers la relation de cette première rencontre, emblématique, entre une jeune indienne indigène, autochtone, et un aventurier européen, allogène et exotique, l'indianité et l'européanité s'affrontent, se pénètrent et se fondent en une identité différente, mixte, mélangée. En découvrant et en s'initiant aux mœurs des indiens Tupis, Diogo Álvares tend à devenir l'un d'entre eux. À l'inverse, en venant en Europe, en France, Paraguaçú se transforme en une européenne. Les conceptions indianistes, nativistes ou indigénistes modernes se nourrissent de cette légende. Ces deux mythes de Caramurú et de Paraguaçú constituent un véritable archétype de l'identité brésilienne. Ils incarnent symboliquement une forme d'intégration mutuelle. Jusqu'à quel point, en ces témoignages, ces personnages d'indiens natifs et d'étrangers européens ne seraient-il pas que des métis, des doubles et des mêmes, des reflets symétriques mais inversés, réciproques, des uns et des autres?

Ces indiens sauvages ne seraient que des européens primitifs déguisés. La remarque a été faite en 1961 par un écrivain et un traducteur français, Georges Raeders, ${ }^{75}$ à propos de la traduction française du Caramurú de José de Santa Rita Durão par Eugène Garay de Montglave. Que ce soit dans le poème épique initial, en portugais, ou dans sa transposition en prose, en français, "les Tupinambas de Santa Rita Durão," observe cet auteur, "sont, sinon toujours des Européens travestis en sauvages à la tête ornée de plumes multicolores, du moins, bien souvent, des indigènes idéalisés" 76 qui vivent, qui combattent et qui meurent comme "de véritables chevaliers [européens ] du Moyen-âge,"77 qui sont soumis à des princes. Ils sont adoubés "par un léger coup de plat d'épée sur l'épaule."78 L'héroïne, Paraguassu, apparaît au moins une fois en une "véritable Amazone, armée à l'européenne, avec une cotte de maille, une épée et un casque." ${ }^{79}$ En d'autres passages, ces indiens imaginaires expriment des sentiments et des idées qu'ils n'auraient pas pu avoir au XVI siècle. C'est le cas au chant III de Caramurú de José de Santa Rita Durão qui correspond aux chapitres 10 à 12 de Caramuru, ou la découverte de Bahia par Eugène Garay de Montglave. Gupeva, le chef de la tribu indienne qui a recueilli Diogo Álvares, expose longuement à son interlocuteur européen ses conceptions métaphysiques, étrangement proches des croyances chrétiennes, comme le relève

75 Georges Raeders (1896-1955), universitaire et écrivain français, professeur à l'université catholique de São Paulo (SP) au Brésil.

76 Georges Raeders, "Sur une traduction française de Caramurú, de Santa Rita Durão." Revista Paideia, Universidade de Sorocaba (SP). Brasil, Volume III, Tome I, 1958, 107 et sq.

77 Raeders, 107 et sq.

78 Raeders, 107 et sq.

79 Raeders, 107 et sq. 
également Georges Raeders. Un autre exemple, encore beaucoup d'autres, ce serait le moment "où Paraguassu," commente ce même critique, "demande après la bataille à Álvares ce que deviennent les âmes des morts non baptisés qu'elle aperçoit. Celui-ci lui expose alors le dogme de la damnation éternelle mais elle répond en argumentant comme un philosophe du XVIII siècle." ${ }^{80} \mathrm{Ces}$ indiens frustes sont très évolués. Sous la plume de José de Santa Rita Durão ce sont des européens civilisés mais déguisés en sauvages primitifs.

En revanche, les étrangers, les Européens, ne seraient que d'autres sauvages mais indiens et civilisés, raffinés, policés. Le choc des cultures est violent. Au chant VII de Caramurú de José de Santa Rita Durão et au chapitre 22 de Caramuru, ou la découverte de Bahia d'Eugène Garay de Montglave, Paraguaçú est comme anéantie par le spectacle de la civilisation à son arrivée en France. Lorsqu'elle remonte la Seine vers Paris, sur une felouque, une barque légère, "Paraguaçú," note l'auteur, "[...] reste immobile et comme anéantie. Elle ne respire plus, elle ne parle plus, son œil est fixe, son visage sans émotion [...]. Une stupeur soudaine lui ravit la voix, l'ouie, la parole et la mémoire."81 Le voyage d'abord, sur ce qui ne pouvait lui apparaître que comme un très grand navire français, celui du capitaine Duplessis, l'accueil, à Paris, tout au long des rues, par une foule en liesse, la Cour et la réception au palais du Louvre, en la présence du roi de France, Henri II, et de la reine Catherine de Médicis, tout la sidère. Lors de la cérémonie du baptême, "Paraguaçú" devient "Catherine." Lalliance, la juxtaposition des deux prénoms voudrait signifier que désormais, dans le récit et dans la légende, la double identité, européenne et chrétienne, indienne et païenne, de la jeune femme deviendrait le signe et l'illustration de la manière dont l'état de civilisée aurait dorénavant supplanté l'état de nature antérieur. La civilisation aurait triomphé. Son baptême, suivi à la fin de l'épopée par un appel à la conversion au christianisme de tous les indiens serait une façon de suggérer, dans la perspective de José de Santa Rita Durão et de ses épigones, que les peuples européens n'auraient fait que précéder les nations indiennes vers un même but, un même idéal, une "même impulsion divine."82 La décision, prise par "Paraguaçú" de devenir "Catherine," en serait l'indice. L'argument est théologique. Il se fonde aussi sur une conviction indigéniste. "Caramurú [de José de Santa Rita Durão] est," rappelle Georges Raeders, "au témoignage de tous les historiens de la littérature brésilienne, le véritable premier manifeste du nativisme"83 et de l'américanisme dans la littérature brésilienne en langue portugaise.

\footnotetext{
$80 \quad$ Raeders, 107 et sq.

81 Durão, tome III, 164-165.

82 Durão, tome III, 143 .

83 Raeders, 107 et sq.
} 
Ces "autres" seraient-ils les "mêmes" ? Ces étrangers si différents en apparence, ces sauvages, si barbares, ne seraient-ils les uns et les autres, que des métis très semblables, des indianistes brésiliens au sang-mêlé, mi-européens et mi-indiens? Les indiens Tupinambas qui sont décrits par José de Santa Rita Durão, par Eugène Garay de Montglave, par Daniel Gavet et Philippe Boucher et par Olga Obry sont assimilés à des natifs européens qui auraient vécu dans des conditions primitives analogues. Á l'inverse, les Européens civilisés, portugais ou français, qui apparaissent en ces récits ne sont peut-être que d'autres indiens, déjà métissés et assimilés, et déguisés en européens. Aux chants VIII et IX du Caramurú de José de Santa Rita Durão et aux chapitres 26 à 31 du roman-poème d'Eugène Garay de Montglave, quand Catarina Álvares Paraguaçú décrit ce qu'elle présente comme l'avenir du Brésil, la jeune indienne "Paraguaçú," achève de se transformer en "Catarina," 84 et en une sorte de souveraine européenne éclairée du XVIII siècle, à l'exemple des impératrices autrichiennes et russes de l'époque, de Marie-Thérèse d'Autriche à Catherine II de Russie. On est en pleine affabulation mais ce procédé, construit sur une superposition d'amalgames et d'équivalences, permet de traduire la conviction, chez José de Santa Rita Durão, qu'une profonde unité aurait existé, en 1781, quand il écrit son poème, entre le royaume du Portugal et sa colonie, le Brésil, que les Brésiliens de l'époque auraient profondément éprouvé le sentiment d'appartenir à la métropole, au Portugal. Un distique, toutefois, traduit la naissance d'un patriotisme indigène, brésilianiste, et annonce la future indépendance du Brésil, acquise seulement en 1822. Eugène Garay de Montglave traduit presque littéralement ces deux vers: "Vi nascer no Brasil acceras cidades/Famoso vice-reis e illustres gentes"/“j'ai vu naître dans le Brésil de magnifiques cités,/briller de fameux chefs." ${ }^{85}$ Ce passage est interprété par le traducteur comme "une singulière prophétie de l'auteur."86 Ce serait prêter beaucoup de prescience à José de Santa Rita Durão. Mais ce poème épique, Caramurú, n'en reste pas moins une sincère profession de foi nativiste, un premier manifeste indianiste brésilien, duel, métis, mi-indien et mi-européen.

En ces textes, en ces reflets réciproques, la perception de l'autre, de la différence et de l'altérité se transforme sous l'effet de "l'invention d'un passé [qui aurait été] partagé." 87 Les indiens barbares n'auraient été que des

\footnotetext{
84 Durão, tome II, 174 .

85 Durão, tome III, 98.

86 Durão, tome III, 98.

87 Michel Riaudel, “Caramuru et la latinité amérindienne." Siléne. Les Lettres francophones, hispanophones, lusophones et la latinité (actes du colloque des 20 et 21 mai 2010). Poitiers:
} 
européens primitifs. Les étrangers, les européens, n’auraient été que des sauvages civilisés. Les uns et les autres ne seraient que les mêmes. Ils seraient tous des "indianistes" brésiliens mais métissés, mélangés. Ils figureraient tous, à des degrés divers, une conception d'une identité mixte, mélangée, duelle et contradictoire, à demi-indienne et à demi-européenne. La légende de Diogo Álvares Correia, dit "Caramurú," et de Catarina Álvares, dite "Paraguaçú," en serait une préfiguration d'une première forme de globalisation et de mondialisation.

\section{Conclusion}

Le Brésil a été découvert le 22 avril 1500 par Pedro Álvares Cabral. A cette date, en 1500, la population indienne, native, était plus que majoritaire. En 1600, un siècle plus tard, les indiens Tupis n'étaient plus qu'une infime minorité. L'indianisme est peut-être né à cette époque, entre 1510 et 1550. Si l'on suit la légende de Diogo Álvares Correia, le premier habitant européen, portugais, du Brésil, et de son épouse indienne Catarina Álvares Paraguaçú, baptisée en France, en $15^{22}$ à l'église de Saint-Malo, en Bretagne, telle que José de Santa Rita Durão le rapporte en 1781 dans son poème épique, Caramurú, traduit ou plutôt transposé en prose, en français, en 1829, par Eugène Garay de Montglave sous le titre de Caramuru, ou la découverte de Bahia, la prise de conscience et la revendication d'une identité indigène, autonome, mixte, serait née de cette première rencontre entre Catarina Paraguaçú, la "Mère du Brési," et Diogo Álvares, le "Caramurú." Les transpositions, les adaptations, en portugais et en français, de cette légende fondatrice dans Jakaré-Ouassou, ou les Tupinambas de Daniel Gavet et de Philippe Boucher en 1830, dans Paraguassú, un opéra de J. O'Kelly et de J. Villeneuve en 1855, dans Moema de Delgado de Carvalho en 1892 et, entre 1942 et 1953, dans Catarina do Brasil: a Índia que descobriu a Europa et Catherine du Brésil, filleule de Saint-Malo d'Olga Obry, et, enfin, en 2001, dans Catarina Paraguaçú. A Mãe do Brasil de Franco Tasso, correspondent à autant d'étapes de la constitution de ce mythe identitaire majeur, fondateur. Cette légende a un fondement historique. Á la fin de son roman, en portugais comme en français, Olga Obry rappelle les rares traces qui ont été retrouvées de l'existence historique de ce couple mythique. Diogo Álvares est enterré au monastère de Jésus à Salvador da Bahia et Catarina Álvares repose en une chapelle de l'église de la Grâce, à Graça, au centre de cette même ville de Salvador da Bahia. Olga Obry reproduit aussi dans son livre le fac-simile de l'acte de

Université de Poitiers, CRLA-Archivos. <http://www.revue-silene.com/f/index.php? $\mathrm{sp}=$ comm\&comm_id=80 $>$, consulté le 28 juillet 2013 . 
baptême de "Katherine du Brésil," daté du 30 juillet 1528. On n'en sait guère plus. "Catherine a-t-elle jamais existé?", se demande même la romancière. "Est-ce bien Paraguaçú qu'elle s'appelait avant son baptême? La vérité dort, enfouie sous la légende," ${ }^{\prime 8}$ conclut-elle. Il reste un mythe identitaire, nativiste ou indianiste, dont les significations morales et politiques initiales se sont peut-être radicalement inversées entre le XVIIIème et le XXIème siècles. En 1781, dans le Caramurú de José de Santa Rita Durão, Catherine et Diogo Álvares ont deux patries, le Brésil et l'Europe, et font allégeance au roi du Portugal. En 1830, dans Jakaré-Ouassou ou les Tupinambas de Daniel Gavet et de Philippe Boucher, la révolte des indiens Tupinambas préfigure les aspirations indépendantistes et nationalistes des Brésiliens, réalisées en 1822. En 1942 et en 1953, des romans, Catarina do Brasil: a Índia que descobriu a Europa et Catherine du Brésil, filleule de Saint-Malo d'Olga Obry. En 1952, Blaise Cendrars ${ }^{89}$ en résume la légende dans un article intitulé Caramurú, contenu dans Le Brésil, des hommes sont venus, ${ }^{90}$ pour constater qu'on ne savait "rien, rien d'autre [de lui], sauf qu'il était là, ce blanc, sorti des bois avec sa parenté indienne, toute une tribu dont il avait pris la tête et qui l'avait surnommé Caramurú."91 Des films brésiliens tournés entre 1970 et 2001, Como gostoso era meu francês (1970) de Nelson Pereira dos Santos et Caramurú. A invenção do Brasil (2001) de Guel Arraes, et un dernier roman, Catarina Paraguaçú. A Măe do Brasil de Franco Tasso, ont fait ressurgir à la fois cette légende et l'indianisme dans la littérature et au cinéma, au Brésil. Entretemps, entre 1922 et 1928, le Manifeste Anthropophage ${ }^{92}$ d'Oswald de Andrade et la Revue d'Anthropophagie avaient invité les intellectuels brésiliens à réfléchir sur l'état de dépendance culturelle qui existait à l'époque au Brésil, à l'égard de l'Europe. Cet "indianisme" culturel, hybride, se nourrit de beaucoup de nostalgie. Il exprime une certaine vérité, ne serait-ce que celle d'une identité brésilienne, indienne et métisse, spécifique. Il s'y mêle certainement beaucoup d'erreurs. Cet effort de reconstruction et de déconstruction de l'histoire inventée et romancée de Catarina Álvares Paraguaçú et de Diogo Álvares Correia s'inscrit à son tour à l'intérieur de leur légende. Le dernier mot de ce mythe fondateur n'est pas encore dit.

\footnotetext{
88 Obry, Catherine du Brésil, 140.

89 Blaise Cendrars (1887-1961), de son vrai nom Frédéric Louis Sauser, journaliste et écrivain d'origine suisse, naturalisé français en 1916.

90 Blaise Cendrars, "Caramurú." Le Brésil, des hommes sont venus (1952). Paris: Fata Morgana, $1987,35^{-65}$.

91 Cendrars, 63.

92 Oswald de Andrade, "Manifesto Antropófago" ["Manifeste Anthropophage"]. Revista de Antropofagia - ["Revue d'Anthropophagie"]. São Paulo (SP), n 1, mai 1928, 3, 7.
} 
Cette effervescence esthétique et intellectuelle, très réduite et minoritaire au Brésil, ne préjuge pas non plus de son véritable impact sur la vie politique brésilienne moderne.

\section{Bibliographie}

\section{Ouvrages}

Abbeville, Claude d'. Histoire de la mission des Pères capucins en lisle de Maragnan et terres ciconvoisines, où est traicté des singularitez admirables et des moeurs merveilleuses des Indiens habitans de ce pays, avec les missives et advis qui ont esté envoyez de nouveau par le R.P. Claude d'Abbeville. Paris: imprimerie de F. Huby, 1614.

Andrade, Mario de. Macunaíma : a herói sem nenhum caráter (Macounaïma: le héros sans aucun caractère, traduction française : 1997). São Paulo (SP): Oficinas Gráficas de Eugenio Cupolo, 26 jul., 1928.

Delgado de Carvalho Joaquim, Torres. Moema, opéra créé au Théâtre lyrique de Rio-de -Janeiro en 1892, s.l., s.r.

Durão, Santa Rita José de. Caramurú. Poema épico do descobrimento da Bahia. Lisbóa (Portugal), na Regia officina typografica, 1781, in-12. Édition en ligne, site Scribd: $<$ http://www.scribd.com/api_user_11797_vivancrisologo/d/7289661-Jose-de-Santa -Rita-Durao-Caramuru-1781>.

Durão, José de Santa Rita. Caramaru, ou la découverte de Bahia [traduit par Eugène Garay de Montglave].Paris: Eugène Renduel, 1829. Édition en ligne, site Gallica de la Bibliothèque Nationale de France:tomeI: <http://gallica.bnffrr/ark:/2148/bpt6k57403818 .r=Santa+Rita+Durao.langFR $>$.tome II: $<$ http://gallica.bnf.fr/ark:/12148/bpt6k5739362f .r=Santa+Rita+Durao.langFR $>$. tome III: <http://gallica.bnf.fr/ark:/12148/bpt6k5740 3959.r=Santa+Rita+Durao.langFR>.

Évreux, Yves d'. Suite de l'histoire des choses plus mémorables advenues en Maragnan ès années 1613 et 1614. Second traité [Texte imprimé]. Paris: F. Huby, MCDXC (sic), édition critique du texte complet par Franz Obermeier, Kiel: Westensee-Verlag, cop. 2012.

Franco, Tasso. Catarina Paraguaçú - A Mãe do Brasil ["Catarina Paraguaçú - la Mère du Brésil"], Rio-de-Janeiro (RJ): Editado pela Relume Dumará, 2001. Édition en ligne, sur le site Google Livre : <http://books.google.fr/books?id=PJMtAAAAYAAJ\&q=Cata rina+Paragua $\% \mathrm{C}_{3} \% \mathrm{~A}_{7} \% \mathrm{C}_{3} \% \mathrm{BA}+\mathrm{A}+\mathrm{M} \mathrm{C}_{3} \mathrm{C}_{3} \mathrm{~A}_{3} \mathrm{e}+\mathrm{do}+$ Brasil, $\& d q=$ Catarina + Para gua $\% \mathrm{C}_{3} \% \mathrm{~A}_{7} \% \mathrm{C}_{3} \% \mathrm{BA} .+\mathrm{A}+\mathrm{M} \% \mathrm{C}_{3} \% \mathrm{~A}_{3} \mathrm{e}+\mathrm{do}+\mathrm{Brasil}, \&$ hl=fr\&sa=X\&ei=oZdcT9iPM MeA8wP-ncyDDw\&ved $=0$ CDoQ6AEwAA $>$.

Gavet, Daniel et Philippe Boucher. Jakaré-Ouassou, ou les Tupinambas. Paris: Timothy Dehay, 1830, 237. Édition en ligne, site Gallica de la Bibliothèque Nationale de 
France : <http://gallica.bnf.fr/ark:/12148/bpt6k56272819.r=Jakar\%C3\%A9-Ouassou+ ou+les+Tupinambas.langFR $>$.

Léry, Jean de. Histoire d'un voyage fait en la terre du Brésil, autrement dit Amérique (Première édition : (s. l.), pour A. Chuppin, 1578, In-12). Édition de Frank Lestringant. Montpellier : Presses du Languedoc/Max Chaleil Éditeur, 1992.

Montaigne, Michel de. Essais, 1580-1587 [traduction en français moderne du texte de l'édition de 1595 par Guy de Pernon]. Leogeats: G. de Pernon, 2008. <Édition en ligne, site Trismégiste $:<\mathrm{http}: / /$ www.bribes.org/trismegiste/montable.htm>.

Obry, Olga. Catarina do Brasil : a Índia que Descobriu a Europa ["Catherine du Brésil : l'indienne qui découvrit l'Europe"]. Rio-de-Janeiro (RJ): Atlantica Editora, 1942. Édition en ligne, site Google Livres: <http://books.google.fr/books/about/ Catarina_do_Brasil_a_\%C3\%ADndia_que_descobri.html?id=S6gXAAAAIAAJ\& redir_esc $=\mathrm{y}>$.

Obry, Olga. Catherine du Brésil, filleule de Saint-Malo. Paris: Nouvelles Éditions Latines, 1948. Édition en ligne, site Google Livres : <http://books.google.fr/books?id= $\mathrm{xYkerLo7DfMC} \& p g=\mathrm{PA} 165 \& \mathrm{lpg}=\mathrm{PA} 165 \& \mathrm{dq}=\mathrm{Bapt} \% \mathrm{C}_{3} \% \mathrm{AAme}+\mathrm{de}+\mathrm{Catherine}+\mathrm{du}+$ $\mathrm{Br} \% \mathrm{C}_{3} \%$ Agsil\&source $=$ bl\&ots $=$ zecupmCVI $5 \&$ sig $=$ YdQeZf8eISK 52 BH8Oadlb $\mathrm{Xvn} \_$J4\&hl=fr\&sa=X\&ei=nJFcT-XjCMXq8QOEyvWvBw\&ved=oCDkQ6AEwAA\# $>$.

O’Kelly, J. et J. Villeneuve. Paraguassú : chronique brésilienne. Paris, Bolle-Lassalle, 1855. Raeders, Georges. "Sur une traduction française de Caramurú, de Santa Rita Durão," Revista Paideia, Universidade de Sorocaba (SP). Brasil: Volume III, Tome I, $195^{8}$.

Staden, Hans. Nus, féroces et anthropophages (1557), traduit de l'allemand par Henri Ternaux Compans. Paris, Métailié, 2005.

Thevet, André. Les Singularitez de la France antarctique, autrement nommée Amérique, \& de plusieurs terres et isles découvertes de nostre temps, Paris: chez les héritiers de Maurice de La Porte, 1558. [Éd. intégrale établie, présentée \& annotée par Frank Lestringant]. Paris: Chandeigne, 1997. Édition en ligne, site Gallica : <http://gallica .bnf.fr/ark:/12148/bpt6k109516t>.

\section{Articles}

Andrade, Oswald de. "Manifesto Antropófago"[«Manifeste Anthropophage »]. Revista de Antropofagia - [«Revue d'Anthropophagie»]. São Paulo (SP), n 1, mai 1928.

Cendrars, Blaise. "Caramurú." Le Brésil, des hommes sont venus. Paris, Fata Morgana, 1987 .

Raeders, Georges. "Sur une traduction française de Caramurú, de Santa Rita Durão." Revista Paideia, Universidade de Sorocaba (SP). Brasil, Volume III, Tome I, $195^{8}$.

Riaudel, Michel. "Caramuru et la latinité amérindienne." Siléne. Les Lettres francophones, hispanophones, lusophones et la latinité (actes du colloque des 20 et 21 mai 2010). Poitiers, Université de Poitiers, CRLA-Archivos. < http://www.revue-silene.com/f/ index.php?sp=comm\&comm_id=80>. 


\section{Filmographie}

Guel, Arraes. Caramurú. A invenção do Brasil (Caramurú. La découverte du Brésil), Globo Filmes-Lereby Productions, 2001.

Santos, Nelson Pereira dos. Como gostoso era meu francês (Qu'il était bon mon petit Français), long métrage, DVD, 1971. 